UDC 316.334.56

LBC 60.56

\title{
LIFE-WORLD OF METROPOLIS: SPECIFICS OF GENDER PERCEPTION
}

\author{
Svetlana A. Ilynykh \\ Novosibirsk State University of Economics and Management, Novosibirsk, Russian Federation
}

\begin{abstract}
The article discusses the view of a metropolis, due to the formation of metropolis identity. This identity is associated with a complex of sociocultural, socio-psychological processes, including the process of socialization in a big city. In a metropolis, patriarchal stereotypes are manifested to a lesser extent than in small cities, which contribute to a more rigid consolidation of gender roles. There is a smoothing of the gender aspects of consciousness. This situation is due to the fact that, on the one hand, the socialization of residents of megacities, as well as residents of other territories, occurs within the framework of gender culture. But, on the other hand, residents of megacities in everyday life are much more likely to encounter practices that go beyond the framework of the traditional socio-gender system. The paper provides empirical examples illustrating the characteristics of a metropolitan identity. Gender smoothing is taking place when evaluating the activities of educational and cultural institutions. Approximately equal shares of men and women assess the activities of educational and cultural institutions as high-level work. The low -level work of educational and cultural institutions is also estimated by men and women in approximately equal proportions. The results of the study show that men living in megacities stand in solidarity with women in their assessments. This may indicate that men and women are equally involved in raising children, which reflects the process of smoothing out gender stereotypes. No gender differences were also found when studying respondents' assessments of certain aspects of the social sphere. It is shown that the gender aspects of consciousness remain when assessing the sphere of health care. Significant differences in assessments of health care institutions exist when studying the question of satisfaction with the quality of state medical services, the availability of medical care and the work of ambulances. It is concluded that the smoothing of the gender aspects of consciousness is caused not only by personal characteristics but also by the individual living in a big city.
\end{abstract}

Key words: megalopolis, megalopolis identity, gender aspects of consciousness, education, culture, healthcare

УДК 316.334 .56

ББК 60.56

\section{ЖИЗНЕННЫЙ МИР МЕГАПОЛИСА: СПЕЦИФИКА ГЕНДЕРНОГО ВОСПРИЯТИЯ}

\author{
Светлана Анатольевна Ильиных \\ Новосибирский государственный университет экономики и управления, \\ г. Новосибирск, Российская Федерация
}

\begin{abstract}
Аннотация. В статье рассматриваются особенности восприятия мегаполиса, обусловленные формированием мегаполисной идентичности. Эта идентичность связана с комплексом социокультурных, социальح. но-психологических процессов, в том числе с процессом социализации в большом городе. В мегаполисе в меньшей мере по сравнению с небольшими городами проявляются патриархальные стереотипы, которые способствуют более жесткому закреплению гендерных ролей. Происходит сглаживание гендерных аспектов сознания. Такая ситуация обусловлена тем, что, с одной стороны, социализация жителей мегаполисов, равно как и жителей других территорий, происходит в рамках гендерной культуры. Но, с другой стороны, жители мегаполисов в повседневной жизни гораздо чаще сталкиваются с практиками выхода за рамки традиционной социогендерной системы. В статье приводятся эмпирические примеры, иллюстрирующие особенности мегаполисной идентичности. Гендерное сглаживание обнаруживается при оценке деятельности образова-
\end{abstract}


тельных и культурных учреждений. Примерно равная доля мужчин и женщин оценивает деятельность образовательных и культурных учреждений как работу на высоком уровне. Низкий уровень работы образовательных и культурных учреждений мужчины и женщины тоже оценивают примерно в равных долях. Результаты исследования показывают, что мужчины, проживающие в мегаполисах, вполне солидарны в оценках с женщинами. Это может свидетельствовать о том, что мужчины и женщины в равной мере участвуют в воспитании детей, что отражает процесс сглаживания гендерных стереотипов. Гендерные различия не обнаружены также и при изучении оценки респондентами некоторых аспектов социальной сферы. Показывается, что лишь при оценке сферы здравоохранения остаются гендерные аспекты сознания. Существенные различия в оценках учреждений сферы здравоохранения имеются при изучении вопроса удовлетворенности качеством государственного медицинского обслуживания, доступности медицинской помощи и работы скорой помощи. Делается вывод о том, что сглаживание гендерных аспектов сознания обусловлено не только личностными особенностями, но и проживанием индивида в большом городе.

Ключевые слова: мегаполис, мегаполисная идентичность, гендерные аспекты сознания, образование, культура, здравоохранение.

Интерес к проблематике города и специфике городского пространства появился у социологов достаточно давно. Сегодня актуальность обращения к исследованию города связана с изучением имплицитных аспектов, оказывающих влияние на качество жизни горожан, на возможность самоактуализации, на практики сохранения здоровья и т. д.

В свое время работы Л. Вирта [Вирт 2016], К. Линча [Линч 1982], А. Лефевра [Лефевр 2002] позволили по-новому взглянуть на жизнедеятельность горожан. Влияние пространства города на его жителей представлено в работах Г. Зиммеля [Зиммель 1996], А. Ф. Филиппова [Филиппов 2000]. Комфортность проживания горожан обоснована в исследовании О.В. Максимчук и Т.А. Першиной [Максимчук, Першина 2014]. Возможности инновационных технологий территориального брендинга рассмотрены в работе И.В. Василенко [Василенко 2016]. Вместе с исследованием города как среды обитания достаточно часто исследуется социологами феномен мегаполиса. И это не случайно, так как городское пространство мегаполисов отличается не только урбанизационными процессами, но и жизненным миром, социальным, социально-экономическим, социокультурным контекстом современности.

Исследование мегаполисов происходит в разрезе социально-демографических, территориальных, экономических и других аспектов урбанизационного процесса. Сразу оговорим, что в рамках данного исследования мы рассматриваем мегаполис евро-атлантического типа с присущими ему типологическими ментальными и социокультурными характери- стиками и не касаемся восточных мегаполисов, где доминирует иной тип ментальности, обусловленный специфичными культурно-историческими особенностями.

Укажем также, что мегаполисы отличаются не только особым типом менталитета мегаполисным, но и тем, что в них в большей мере сглаживаются гендерные различия восприятия благодаря формированию мегаполисной идентичности. Эта идентичность связана с комплексом социокультурных, социальнопсихологических процессов, в том числе с процессом социализации в большом городе [Ilinykh, Udaltsova 2014; Vogelsang 2014]. Здесь в меньшей мере по сравнению с небольшими городами проявляются патриархальные стереотипы, которые способствуют более жесткому закреплению гендерных ролей. Возникает следующая ситуация. С одной стороны, социализация жителей мегаполисов, равно как и жителей других территорий, происходит в рамках гендерной культуры. Но, с другой стороны, жители мегаполисов в повседневнсти гораздо чаще сталкиваются с практиками выхода за рамки традиционной социогендерной системы. Все это так или иначе приводит к сглаживанию гендерных аспектов сознания. Кроме того, существенную роль начинает играть специфика собственно мегаполисного пространства. К примеру, большое значение в восприятии мегаполиса приобретает культурная нелокальность мегаполисного пространства. При восприятии современного мегаполиса преодолеваются свойственные городской организации замкнутость, четкая локализация и маркировка социокультурного и социоментального пространства. Доминирую- 
щими параметрами мегаполиса становятся идеи разомкнутости социокультурного пространства, утраты в мегаполисе имманентной для топографии города оппозиции «центр периферия». Мегаполис отличается от города способностью развиваться и быть на передовом крае культуры, науки, производства и т. Д.

Проиллюстрируем эти идеи результатами эмпирического исследования, проведенного научным коллективом под руководством автора статьи в июне 2018 г. в г. Новосибирске. В исследовании приняли участие 1204 респондента: 542 мужчины и 662 женщины. Выборка сформирована посредством трехступенчатого отбора территорий.

Респондентам в открытом вопросе было предложено оценить черты города Новосибирска, делающие его привлекательным. И мужчины, и женщины (по 13,9 \%) отметили, что это современный развивающийся город. По $8 \%$ респондентов в каждой гендерной группе назвали инфраструктуру, а 7,8 \% в каждой группе - культуру.

Для исследования гендерного восприятия мегаполиса мы задали также ряд вопросов, касающихся качества предоставления услуг в разных сферах жизни горожанина.

Так, респондентам предлагалось оценить качество работы различных учреждений образовательных, культурных, медицинских, учреждений социальной сферы. Шкала оценивания включала в себя такие индикаторы, как «очень высокое», «высокое», «среднее», «низкое», «очень низкое».

Было выявлено, что примерно одинаковая доля опрошенных мужчин и женщин по всем индикаторам высказывается о качестве работы школ, детских учреждений, домов культуры, учреждений дополнительного образования (табл. 1).

Иными словами, примерно равная доля мужчин и женщин оценивает деятельность образовательных и культурных учреждений как работу на очень высоком уровне, на высоком и т. д. Показательно, что и низкий уровень работы образовательных и культурных учреждений мужчины и женщины оценивают примерно одинаково. Эти данные позволяют говорить о том, что происходит сглаживание гендерной специфики восприятия. Если обратиться к стереотипам гендерной культуры, то одной из приоритетных сфер женщины является воспитание детей. Поэтому именно она в большей мере может адекватно оценить деятельность образовательных и культурных учреждений. Однако результаты исследования показывают, что мужчины, проживающие в мегаполисах, вполне солидарны в оценках с женщинами. Это может свидетельствовать о том, что мужчины и женщины в равной мере участвуют в воспитании детей, что отражает процесс сглаживания гендерных стереотипов.

Гендерные различия не обнаружены также и при изучении оценки респондентами некоторых аспектов социальной сферы. Так, при оценке работы общественного транспорта мужчины и женщины высказались почти единодушно о своей неудовлетворенности его работой (3,2 и 3,5 \% соответственно), удовлетворенности в средней степени (34,5 и 28,8 \% соответственно), удовлетворенности (35,5 и 37,7\%), неудовлетворенности (15,1 и 16,6 \%).

Аналогичную картину можно наблюдать и в оценке органов социальной защиты и обес-

Таблицุа 1

Распределение ответов на вопрос об оценке качества работы учреждений, \%

\begin{tabular}{|c|c|c|c|c|c|c|c|c|}
\hline \multirow[t]{2}{*}{ Варианты ответов } & \multicolumn{2}{|l|}{ Школы } & \multicolumn{2}{|c|}{ Детские сады } & \multicolumn{2}{|c|}{ Дома культуры } & \multicolumn{2}{|c|}{$\begin{array}{l}\text { Учреждения } \\
\text { дополнительного } \\
\text { образования }\end{array}$} \\
\hline & $\mathrm{M}$ & Ж & $\mathrm{M}$ & Ж & $\mathrm{M}$ & Ж & $\mathrm{M}$ & Ж \\
\hline 1 - Очень низкое & 2,6 & 1,2 & 2,8 & 1,5 & 2,8 & 2,6 & 3,2 & 2,8 \\
\hline $2-$ Низкое & 2,2 & 4,0 & 4,3 & 4,4 & 5,0 & 4,0 & 3,4 & 4,1 \\
\hline 3-Среднее & 18,4 & 23,0 & 20,5 & 22,5 & 21,1 & 17,9 & 14,0 & 15,3 \\
\hline 4 - Высокое & 44,3 & 43,6 & 35,6 & 39,1 & 29,1 & 37,8 & 32,0 & 34,6 \\
\hline 5 - Очень высокое & 14,2 & 13,7 & 14,6 & 14,6 & 23,9 & 20,9 & 26,4 & 23,7 \\
\hline Затрудняюсь & 18,2 & 14,5 & 22,2 & 17,9 & 18,1 & 16,1 & 21,0 & 19,6 \\
\hline Итого & 100,0 & 100,0 & 100,0 & 100,0 & 100,0 & 100,0 & 100,0 & 100,0 \\
\hline
\end{tabular}


печения. Удовлетворены в средней степени их работой $22,3 \%$ мужчин и 24,8 \% женщин, удовлетворены - 22,8 \% мужчин и 19,3\% женщин, полностью удовлетворены - 5,8 \% мужчин и $8,9 \%$ женщин.

Однако результаты исследования работы медицинских учреждений позволяют выявить небольшие различия в крайних оценках мужчин и женщин. Здесь можно видеть в целом более низкие оценки по сравнению с другими учреждениями. Так, об очень высоком уровне работы больниц высказываются $8,3 \%$ мужчин и $3,5 \%$ женщин, высоком уровне - 24,8 \% мужчин и 20,9\% женщин, среднем уровне - 35,7 \% мужчин и $39,9 \%$ женщин, низком уровне - 19,7 \% мужчин и $20,4 \%$ женщин, очень низком - 6,8 \% мужчин и $11,1 \%$ женщин. Как видим, мужчины чуть чаще указывают высокий и очень высокий уровни и чуть реже - низкий и очень низкий уровни.

Примерно схожая картина наблюдается и при оценке поликлиник. При этом примерно вдвое реже женщины оценивают работу этих учреждений на очень высоком уровне (4\% против $8 \%$ ).

Такие гендерные различия, вероятно, можно объяснить тем, что мужчины по сравнению с женщинами в меньшей мере пользуются услугами медицинских учреждений.

Еще большие различия в оценках учреждений сферы здравоохранения мы получили на вопрос об удовлетворенности качеством государственного медицинского обслуживания, доступности медицинской помощи и работе скорой помощи (табл. 2).
Если оценки «удовлетворен в средней степени», «удовлетворен», «не удовлетворен» у мужчин и женщин близки, то в крайних вариантах ответов - «полностью удовлетворен» и «совсем не удовлетворен» - можно видеть большой разрыв. Особенно он наблюдается при оценке работы учреждений государственного медицинского обслуживания. Женщины почти втрое чаще высказываются в негативном ключе.

Полученные результаты позволяют говорить о том, что женщины более критично оценивают работу учреждений здравоохранения. Это, возможно, связано с тем, что они в большей мере воспринимают свою ответственность не только за собственное здоровье, но и за здоровье своих близких.

Дополняют полученные выводы результаты исследования, проведенного в 2019 г. под руководством автора. В нем приняли участие 372 мужчины и 400 женщин. Тип выборки трехступенчатая. Хотя тема исследования была полностью посвящена состоянию здравоохранения в городе Новосибирске, некоторые результаты отражают и элементы мегаполисного менталитета, и их гендерную специфику. Так, элементами мегаполисного менталитета можно назвать более критичное отношение ко многим социальным процессам, в том числе и в здравоохранении. Такое критичное отношение обусловлено тем, что жители мегаполиса воспринимают свою бытийность не только как жизнь в городе, но как жизнь в крупном городе, мегаполисе, где, соответственно, должны быть и более комфортные условия проживания, и более высокое каче-

Таблица 2

\section{Распределение ответов на вопрос об удовлетворенности качеством работы} учреждений сферы здравоохранения, \%

\begin{tabular}{|c|c|c|c|c|c|c|}
\hline \multirow[t]{2}{*}{ Варианты ответов } & \multicolumn{2}{|c|}{$\begin{array}{l}\text { Качество } \\
\text { государственного } \\
\text { медицинского } \\
\text { обслуживания }\end{array}$} & \multicolumn{2}{|c|}{$\begin{array}{l}\text { Доступность } \\
\text { медицинской } \\
\text { помощи }\end{array}$} & \multicolumn{2}{|c|}{$\begin{array}{l}\text { Работа скорой } \\
\text { помощи }\end{array}$} \\
\hline & $\mathrm{M}$ & Ж & $\mathrm{M}$ & Ж & $\mathrm{M}$ & Ж \\
\hline Совсем не удовлетворен & 6,9 & 19,4 & 7,1 & 14,8 & 5,7 & 11,9 \\
\hline Не удовлетворен & 21,4 & 24,3 & 19,7 & 21,5 & 15,1 & 15,4 \\
\hline $\begin{array}{lll}\text { Удовлетворен } & \text { в } & \text { средней } \\
\text { степени } & & \\
\end{array}$ & 38,2 & 35,1 & 34,2 & 38,0 & 30,8 & 26,6 \\
\hline Удовлетворен & 25,3 & 17,3 & 29,2 & 19,7 & 25,8 & 26,6 \\
\hline Полностью удовлетворен & 5,2 & 2,0 & 7,8 & 4,3 & 10,9 & 7,6 \\
\hline Затрудняюсь & 2,9 & 2,0 & 2,1 & 1,8 & 11,7 & 11,9 \\
\hline Итого & 100,0 & 100,0 & 100,0 & 100,0 & 100,0 & 100,0 \\
\hline
\end{tabular}


ство жизни. Одним из элементов качества жизни является сфера здравоохранения. Критичное отношение мы выявили во многих аспектах, касающихся данной сферы. В статье приведем лишь некоторые, наиболее полно отражающие мегаполисный менталитет и гендерную специфику.

Например, 41,1 \% мужчин недовольны тем, что для приема нужного специалиста нужно совершить такое «ненужное действие», как посетить терапевта. По существу это целесообразное действие, позволяющее упорядочить обращения к узким специалистам. Но мегаполисный менталитет не позволяет его оценить в полной мере. Правда, в группе женщин только 35,3 \% опрошенных высказались негативно в отношении установленного правила - приоритетного посещения терапевта.

Еще одним показателем, иллюстрирующим мегаполисный менталитет, является поиск другого врача, если, по мнению опрошенных, им был поставлен неверный диагноз. Стоит заметить, что вообще особенностью россиян является самостоятельная постановка диагноза. Но если сельский житель чаще всего обратится к народным средствам, то житель мегаполиса - к другому врачу (55,6 \% мужчин и $60,8 \%$ женщин). И крайняя мера обращение в суд при нанесении вреда здоровью - тоже характерна в большей мере для жителей мегаполиса. Следует сказать, что к этой мере жители мегаполиса обращаются крайне редко - 4,6 \% мужчин и 5,7 \% женщин. Таким образом, мы видим и некоторые гендерные различия, и сглаживания одновременно при оценке здравоохранения.

Подведем итоги. Жизненный мир мегаполиса имеет свою специфику. Она проявляется во множестве феноменов. Одним из таковых является то, что мегаполис формирует особый тип идентичности - мегаполисную идентичность. Она отличается от традиционно-городской идентичности. Эти отличия обусловлены не только личностными особенностями, но и проживанием индивида в большом городе, способностью мегаполиса к быстрому развитию, способностью быть на передовом крае культуры, науки, а также тем, что происходит сглаживание гендерных аспектов сознания. Результаты эмпирического исследо- вания позволяют подтвердить эти особенности мегаполисного сознания. При этом отметим, что гендерные аспекты сознания продолжают оставаться, особенно в отношении тех сфер деятельности горожан, которые являются жизненно важными.

\section{СПИСОК ЛИТЕРАТУРЫ}

Василенко 2016 - Василенко И.А. Возможности инновационных технологий территориального брендинга для формирования современного имиджа российских регионов // Власть. 2016. № 1. C. 68-73.

Вирт 2016 - Вирт Л. Урбанизм как образ жизни: пер. с англ. М.: Strelka Press, 2016.

Зиммель 1996 - Зиммель Г. Социология пространства // Избранное. В 2 т. Т. 2. Созерцание жизни. М.: Юристь, 1996.

Максимчук, Першина 2014-Максимчук О.В., Першина T.A. Оценка уровня и качества жизни горожан с позиций комфортности проживания в современном городе (на примере крупных городов ЮФО) // Социология города. 2014. № 2. C. 33-35.

Линч 1982 - Линч К. Образ города. М.: Стройиздат, 1982.

Лефевр 2002 - Лефевр А. Идеи для концепции нового урбанизма // Социологическое обозрение. 2002. Т. 2, № 3. С. 19-26.

Филиппов 2000 - Филиппов А.Ф. Социология пространства // Логос. № 2 (23). С. 113-151.

Ilinykh, Udaltsova 2014-Ilinykh S.A., Udaltsova M.V. Gender aspects of entrepreneurship in Russia // Ecology, Environment and Conservation. 2014. Vol. 20. P. 499-505.

Vogelsang 2014 - Vogelsang $F$. Identität in einer offenen Wirklichkeit. Eine Spurensuche im Anschluss an Merleau-Ponty, Ricoeur und Waldenfels. Freiburg / München: Verlag Karl Alber, 2014.

\section{REFERENCES}

Vasilenko I.A., 2016. Possibilities of innovative technologies of territorial branding for the formation of a modern image of Russian regions. Vlast, no. 1, pp. 68-73.

Wirth L., 2016. Urbanism as a way of life. Moscow, Strelka Press.

Simmel G., 1996. The Sociology of Space. Favorites. In 2 vols. Vol. 2. Contemplation of life. Moscow, Yurist Publ. 


\section{СОЦИОЛОГИЯ И СОЦИАЛЬНЫЕ ТЕХНОЛОГИИ}

Maksimchuk O.V., Pershina T.A., 2014. Assessing the level and quality of life of citizens from the standpoint of comfortable living in a modern city (by the example of large cities of the Southern Federal District). Sotsiologiya goroda, no. 2, pp. 33-35.

Lynch K.,1982. The image of the city. Moscow, Stroyizdat Publ.

Lefebvre A., 2002. Ideas for the concept of a new urbanism. Sotsiologicheskoe obozrenie, vol. 2, no. 3, pp. 19-26
Filippov A.F., 2000. Sociology of space. Logos, no. 2 (23), pp. 113-151.

Ilinykh S.A., Udaltsova M.V., 2014.Gender aspects of entrepreneurship in Russia. Ecology, Environment and Conservation, vol. 20, pp. 499-505.

Vogelsang F., 2014. Identität in einer offenen Wirklichkeit. Eine Spurensuche im Anschluss an Merleau-Ponty, Ricoeur und Waldenfels. Freiburg, München, Verlag Karl Alber.

\section{Information About the Author}

Svetlana A. Ilynykh, Doctor of Sciences (Sociology), Professor, Head of Department of Sociology, Novosibirsk State University of Economics and Management, Kamenskaya St., 52, 630099 Novosibirsk, Russian Federation, ili.sa@mail.ru, https://orcid.org/0000-0001-7109-4292

\section{Информация об авторе}

Светлана Анатольевна Ильиных, доктор социологических наук, профессор, заведующая кафедрой социологии, Новосибирский государственный университет экономики и управления, ул. Каменская, 52, 630099 г. Новосибирск, Российская Федерация, ili.sa@mail.ru, https://orcid.org/0000-0001-7109-4292 